\title{
State and trait anxiety related gamma oscillations in patients with anxiety within the Research Domain Criteria (RDoC) framework
}

Kyoung Min Kim

Dankook University College of Medicine

Su Hyun Bong

Daegu Catholic University School of Medicine

Jun Won Kim ( $\boldsymbol{D}$ f_affection@naver.com )

Daegu Catholic University School of Medicine

\section{Research Article}

Keywords: anxiety, trait, state, quantitative electroencephalography, resting state

Posted Date: September 8th, 2021

DOI: https://doi.org/10.21203/rs.3.rs-775299/v1

License: (c) (i) This work is licensed under a Creative Commons Attribution 4.0 International License.

Read Full License 


\section{Abstract \\ Background}

Diagnosis of anxiety has relied primarily on self-report. This study examined using quantitative electroencephalography (qEEG) to assess the association between anxiety and underlying neural correlates.

\section{Methods}

A total of 41 participants who visited a psychiatric clinic underwent resting state EEG and completed the State-Trait Anxiety Inventory. The absolute power of six frequency bands were analyzed: delta $(1-4 \mathrm{~Hz})$, theta $(4-8 \mathrm{~Hz})$, alpha $(8-10 \mathrm{~Hz})$, fast alpha $(10-13.5 \mathrm{~Hz})$, beta $(13.5-30 \mathrm{~Hz})$, and gamma $(30-80 \mathrm{~Hz})$.

\section{Results}

State anxiety scores were significantly negatively correlated with absolute gamma power in frontal (Fz, $r$ $=-0.484)$ and central $(C z, r=-0.523)$ regions, while trait anxiety scores were significantly negatively correlated with absolute gamma power in frontal $(F z, r=-0.523)$, central $(C z, r=-0.568)$, parietal $(P 7, r=$ $-0.500 ; \mathrm{P} 8, r=-0.541)$, and occipital $(01, r=-0.510 ; 02, r=-0.480)$ regions.

\section{Conclusions}

The present study identified the significantly negative correlations between the anxiety level and gamma band power in fronto-central and posterior regions assessed at resting status. Further studies to confirm our findings and identify the neural correlates of anxiety are needed.

\section{Background}

The Diagnostic and Statistical Manual of Mental Disorders (DSM), which is a widely used classification system of mental health and disease, makes diagnostic deductions based on symptoms and behaviors reported by patients or observed by clinicians [1]. However, this diagnostic system is limited in that individuality is lost in evaluation, treatment, and prognosis because heterogeneously expressed symptoms are categorized as a part of a single disease entity. For instance, the diagnostic criteria for major depressive disorder in the DSM-5 includes nine heterogeneous symptoms: depressed mood, diminished interest, sleep disturbances, psychomotor agitation or retardation, fatigue, worthlessness, cognitive dysfunction, and suicidal ideation [1]. In addition, although the symptom presentation of patients might vary widely, the diagnosis of major depressive disorder depends on counting the number of symptoms, which might result in lack of consideration of individuality in the evaluation and treatment process. 
Another limitation of classic diagnostic systems in psychiatry, unlike other fields of medicine, is a lack of incorporating neuroscientific technology developed in recent decades for diagnostic purposes [2]. Reflecting the concerns for these limitations, the DSM-5 adopted a dimensional approach regarding psychiatric disorders, viewing them as spectral entities rather than as strictly categorical, and new research results have been reported based on this revised classification system [1].

Besides the changes in diagnostic systems, the Research Domain Criteria (RDoC) project was proposed by the National Institute of Mental Health in 2010 as a framework to integrate information from various sources and domains, including symptoms, genetics, neuroscience, and physiology, as applied to understanding and conducting research regarding psychiatric disorders. RDoC is not a new classification system replacing the DSM, but a framework for the research of mental health and disease. RDoC includes six research areas (negative valence, positive valence, cognitive systems, systems for social processes, arousal/regulatory systems, and sensorimotor systems), with research being actively conducted worldwide [3]. For example, with respect to anxiety, there is an RDoC Anxiety and Depression project (RAD project), in which brain imaging has been used to establish and explain the association between brainbased constructs and anxiety symptoms [4].

Anxiety, one of the most common psychopathologies, is a complex physical and psychological emotional response. It is an unpleasant psychological state in which one might experience tension and irritability with the anticipation of a future threat [1]. It is often accompanied by various physiological responses of the autonomic nervous system, such as tachycardia, tremor, and dizziness as well as muscle tension and vigilance [1]. To assess the severity of anxiety symptoms in patients, the State-Trait Anxiety Inventory (STAI) is one of the most widely used self-reporting checklists for the assessment of anxiety symptoms [5]. The STAI consists of two subdomains: state anxiety evaluates the intensity of current feelings "at this moment," and trait anxiety assesses the proneness to anxiety as a temperamental trait [5]. Although both share common features in that they assess the anxiety of an individual, they are reported to have some distinct neurobiological characteristics. For instance, a functional imaging study reported that while the anterior insula and basolateral amygdala constitute a network linked to both, state and trait anxiety are distinctively linked to dynamic functional and more static structural neural aspects, respectively [6]. Differences between state and trait anxiety are also observed in clinical tasks assessing the performance of executive function. [7] reported that higher trait anxiety predicted lower performance in a Stroop task evaluating executive function whereas higher state anxiety was associated with better performance.

The assessment of anxiety has relied primarily on self-report. However, in recent years, there have been increasing reports that electroencephalography (EEG) might be helpful in the assessment of the mental health disorders $[8,9]$. In particular, quantitative electroencephalography (qEEG) is a neurological technique that analyzes the spectral band power of the delta $(1-4 \mathrm{~Hz})$, theta $(4-8 \mathrm{~Hz})$, alpha $(8-13 \mathrm{~Hz})$, beta $(13.5-30 \mathrm{~Hz})$, and gamma $(30-80 \mathrm{~Hz})$ frequency bands. qEEG has several benefits over other neuroimaging techniques, in that it is non-invasive with no radiation exposure, it has excellent time resolution, and a low inspection cost $[10,11]$. While qEEG can assist the physician in making a diagnosis, it can do more than simply detecting EEG abnormalities and helping form a diagnosis [11]. Specifically, 
qEEG can define subtypes of a disorder by identifying differential pathophysiological processes in patients who present with similar clinical symptoms $[11,12]$. Its use in this way has been replicated in the assessment of various psychiatric disorders, such as providing probability estimates of the likelihood that a child may have a learning or attentional disorder [11, 12]. qEEG might also help in differentiating the categories of dementing disorders such as Alzheimer's disease, vascular dementia, alcoholism, and delirium [11].

qEEG has also been applied in studies investigating disorders that are associated with anxiety, such as panic, posttraumatic stress (PTSD), and obsessive-compulsive disorders (OCD) $[9,13,14]$. For instance, studies have reported diminished alpha activity in anxiety disorder [15-17] and increased theta activity in OCD $[18,19]$. However, compared to other psychiatric disorders, the literature on qEEG and anxiety disorder is small and difficult to translate to the clinical setting [11] due to the heterogeneity of symptoms among the disorders included in anxiety disorders [20].

Thus, the present study aimed to investigate the neural correlates of anxiety with qEEG focusing on the state and trait anxiety defined according to the RDoC framework existing across the differential diagnosis, rather than focusing on the diagnosis. In pursuing this purpose, we used the self-reporting of outpatients to examine anxiety symptoms and the various factors that influence it, such as sociodemographic factors and depressive symptoms. After controlling for these factors, we expected to find that anxiety symptoms were independently related to qEEG.

\section{Methods}

\section{Participants}

Individuals who visited the psychiatric clinic at Daegu Catholic University Hospital from 2017 to 2018 were considered for inclusion in the study. The inclusion criteria were individuals with anxiety as the main symptom and between 19 and 70 years old. Individuals with brain damage, a neurological disorder, a genetic disorder, substance dependence, epilepsy or any other mental disorder reported during a personal history and anamnesis were excluded from participation in this study. Individuals who exhibited an IQ of 70 or lower according to the Wechsler Adult Intelligence Scale, 4th edition, Korean version (K-WAIS-IV) or who needed immediate intervention due to the possibility of self-harm and aggressive behavior were also excluded.

\section{Depression assessment}

The Beck Depression Inventory-II (BDI-II) measures the degree of severity of clinical depression symptoms [21]. It consists of 21 items scored on a scale from 0 to 3; items include cognitive, emotional, motivational, and somatic symptoms. The total score ranges from 0 to 63 , and a higher total score means more severe depressive symptoms. In this study, we used the scale validated by Sung et al. [22].

\section{Anxiety assessment}


The STAI assesses anxiety in adults [5] and consists of two self-rating 20-items rated by 4 point-Likert scale, one measuring state anxiety and the other measuring trait anxiety. The state anxiety scale requires people to describe how they feel in the present. State anxiety scores increase in response to situational stress and decline under relaxed conditions. The trait anxiety scale asks people to indicate how they generally feel. Trait anxiety scores reflect relatively stable individual differences in anxiety proneness that are impervious to situational stress. This study used the Korean translation of the STAI from Kim [23], which has previously been shown to exhibit excellent psychometric properties, with an internal consistency reported at Cronbach $a=0.91$ [24].

\section{EEG recording and pre-processing}

This study's methods ('EEG recording, pre-processing, power spectrum analysis, and statistical analysis') were the same as the core methodology used in the authors' previous studies [25-27]. In the past few years, the authors have studied the use of qEEG as a diagnostic marker for psychiatric disorders as described in the DSM-5, such as attention-deficit hyperactivity disorder and schizophrenia, using the same research methodology as in this study. Here, the existing EEG protocol was used to study the relationship between qEEG and anxiety symptoms in the RDoC framework.

The EEG recordings were performed using a SynAmps2 direct-current (DC) amplifier and a 10-20 layout 64-channel Quick-Cap electrode placement system (Neuroscan Inc., NC, USA). The EEG data were digitally recorded from 21 gold cup electrodes placed according to the international 10-20 system. The impedances were maintained below $5 \mathrm{k} \Omega$, and the sampling rate was $1000 \mathrm{~Hz}$. We used the linked mastoid reference and two additional bipolar electrodes to measure the horizontal and vertical eye movements.

Matlab 7.0.1 (Math Works, Natick, MA, USA) and the EEGLAB toolbox [28] were used to pre-process and analyze the EEG recordings. First, the EEG data were downsampled to $250 \mathrm{~Hz}$. Next, the EEG data were detrended and mean-subtracted to remove the DC component. A 1- $\mathrm{Hz}$ high-pass filter and a $60-\mathrm{Hz}$ notch filter were applied to remove the eye and electrical noise. Next, independent component analysis (ICA) was performed to remove well-defined sources of artifacts. ICA has been demonstrated to reliably isolate artifacts caused by eye and muscle movements and heart noise [29]. Components that corresponded to eye blinking or muscle movement were identified using a published technique that compares favorably with other artifact rejection techniques [30]. We identified and removed at least one component that corresponded to muscle artifacts, and no detected residual muscle artifacts. Finally, clinical psychiatrists and EEG experts visually inspected the corrected EEGs. For the analysis, we selected more than two minutes of artifact-free EEG readings from the three-minute recordings.

\section{Power spectrum analysis of the EEG recordings}

Six frequency bands were defined for further analysis: delta $(1-4 \mathrm{~Hz})$, theta $(4-8 \mathrm{~Hz})$, slow alpha (8-10 $\mathrm{Hz})$, fast alpha $(10-13.5 \mathrm{~Hz})$, beta $(13.5-30 \mathrm{~Hz})$, and gamma $(30-80 \mathrm{~Hz})$. The spectral power of the EEG data was calculated via fast Fourier transformation using the "spectrogram.m" function of the signal processing toolbox in Matlab. Time windows of 1000-ms with an $800-\mathrm{ms}$ overlap and Hamming window 
were used for the spectral analysis. Finally, the absolute powers were averaged over all of the time windows and frequency bands for further analysis.

\section{Statistical analyses}

All of the values are expressed as mean and standard deviation (SD). To assess the relationship between the state and trait anxiety scores and EEG recordings, we used a Pearson's partial correlation analysis that controlled for age and gender. Statistical significance was defined as $p<0.0026(0.05 / 19)$. To control for false positives from multiple comparisons, we used the Bonferroni correction in which the $p$-values were multiplied by the number of comparisons. To improve clarity, topographical plots of the results of the Pearson's partial correlations are presented. All data were analyzed using the Statistical Package for the Social Sciences (SPSS) software, version 18.0 (SPSS Inc., Chicago, IL, USA).

\section{Results}

\section{Demographic and clinical data}

Forty-one individuals ( 11 male and 30 female) voluntarily agreed to participate after receiving an explanation of the purpose and methodology of the study. The mean age of the participants was $53.00 \pm$ 13.56 years for males and $52.53 \pm 11.09$ years for females. The average score of BDI-II for all participants was $31.10 \pm 12.52$, which indicates that participants experience moderate to severe depressive symptoms. In case of the scores of state anxiety and trait anxiety, the average scores were $59.63 \pm 11.74$ and $59.54 \pm 10.86$, respectively, indicating mild to moderate symptoms of anxiety. Demographic and clinical data are summarized in Table 1. 
Table 1

Demographic data and cognitive function of subjects

\begin{tabular}{|ll|}
\hline Mean \pm SD & All subjects $(\mathbf{n}=\mathbf{4 1})$ \\
\hline Gender, $\mathrm{n}(\%)$ & $11(26.8)$ \\
\hline Male & $30(73.2)$ \\
\hline Female & \\
\hline Age & $53.00 \pm 13.56$ \\
\hline Male & $52.53 \pm 11.09$ \\
\hline Female & $31.1 \pm 12.52$ \\
\hline BDI-II & $59.63 \pm 11.74$ \\
\hline STAl (state) & $59.54 \pm 10.86$ \\
\hline STAl (trait) & \\
\hline $\begin{array}{l}\text { Note: SD, Standard deviation; BDI-II, Beck Depression Inventory-II; STAl, Korean version of State Trait } \\
\text { Anxiety Inventory }\end{array}$ \\
\hline
\end{tabular}

\section{Correlation analysis}

Pearson's partial correlation analyses were performed on the state and trait anxiety scores and EEG recordings. We found that the STAI scores were negatively correlated with the absolute gamma power. After applying the Bonferroni correction $(p<0.0026)$, significant findings were revealed in both state and trait anxiety. First, state anxiety scores were significantly negatively partially correlated with the absolute gamma power in the frontal $(F z, r=-0.484)$ and central $(C z, r=-0.523)$ regions. Second, trait anxiety scores were significantly negatively partially correlated with the absolute gamma power in frontal ( $F z, r=$ -0.523), central (Cz, $r=-0.568)$, parietal (P7, $r=-0.500 ; \mathrm{P} 8, r=-0.541)$, and occipital $(01, r=-0.510 ; 02, r=$ -0.480 ) regions. The partial correlations between the STAI scores and the absolute delta, theta, alpha, and beta powers were not statistically significant. The results of Pearson's partial correlation analysis controlling for age and gender between the STAI scores and absolute gamma power are presented in Table 2. The scatter plots and topographical features of the partial correlation are presented in Fig. 1. 
Table 2

The results of the Pearson's partial correlation analysis (corrected for age and gender) between STAI and absolute gamma power

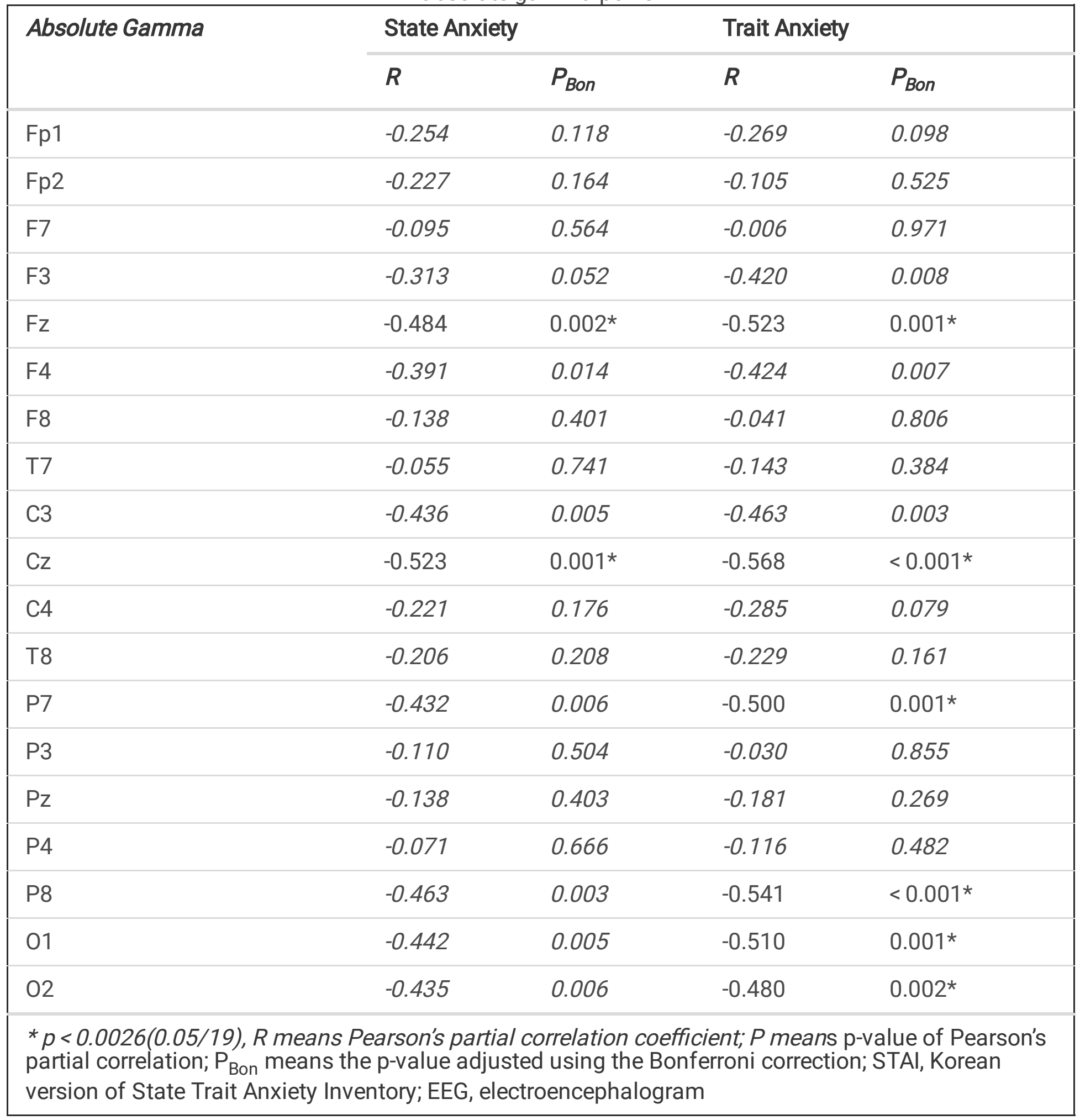

\section{Discussion}

The present study investigated the association between EEG band power and anxiety, both state and trait anxiety, as defined by the STAI. The significant negative correlations between anxiety level and gamma 
band power in fronto-central and posterior regions were the main findings of our study. These findings seem to be contrary to the results from previous studies, as discussed later.

Gamma oscillations, representing the high frequency band $(30-80 \mathrm{~Hz})$, are short-lived and generated from the recurrent coordinated interactions of excitatory and inhibitory neurons. [31-33]. Gamma band activity is the ubiquitous phenomenon of cortical process rather than activity of some specific neurons [33], and reflects various cognitive process such as attention, binding, object representation, memory, and language, among which, memory seems to be the most prominent and fundamental to other cognitive process [33-35]. Particularly, gamma oscillations are related to emotional memory via cortical arousal induced by an emotionally arousing stimulus, which in turn facilitates the coordination of signaling between brain regions, essential for the encoding and expression of emotional memory [36].

Many previous studies have reported that emotional stimuli, especially negative stimuli, induce enhanced gamma activity [36]. For instance, [37] assessed gamma band activity in healthy university students when an emotional stimulus was presented. Participants had enhanced gamma band activity in response to aversive stimuli but no difference when shown neutral pictures. [38] also assessed the gamma band activity of 21 adults when an emotional stimulus was presented, and observed enhanced gamma band activity in the amygdala, as well as in visual, prefrontal, parietal, and posterior cingulate cortices when shown emotional stimuli compared to neutral stimuli.

Among the negative emotions associated with the change of qEEG, some researchers have specifically focused on anxiety, which can be induced in response to particular tasks. [39] investigated the amplitude of the EEG power band oscillation while people with high- and low- math anxiety were expecting an arithmetic problem to be presented. People with high-math anxiety showed stronger gamma band activity, as well as beta band activity, than those with low-math anxiety while solving the arithmetic problem, indicating that individuals with high-math anxiety might use more attentional resources during the anticipation of arithmetic problems. [40] also analyzed EEG band power during the processing of emotional faces as related to state anxiety and reported enhanced gamma band activity in the visual cortex and fusiform gyrus during the presentation of fearful faces. Moreover, an individual's level of state anxiety was positively correlated with the gamma band responses. [41] investigated the change of EEG gamma band power in individuals with high- and low-social anxiety while waiting to give a speech. EEG gamma power increased in the parietal lobe during the speech anticipation period in both groups. However, these increases of gamma power did not differentiate the high- and low-anxiety groups, which suggests that the increased gamma power appears to reflect a state-dependent increase of arousal. Similarly, [42] inspected the level of gamma activity in patients with generalized anxiety disorder and controls during a worry induction task and reported increased gamma activity during the worry induction in both groups.

The previous studies consistently reported increased gamma activity when experiencing negative emotions, including anxiety [36]. Those reports seem to contradict our current findings, in which we found negative associations between gamma power activity and anxiety scores. However, we should consider 
one critical difference between the previous studies and the current. The previous studies evaluated the gamma band activity during an induction of the negative emotion whereas the present study assessed qEEG during resting status. The aforementioned studies [40-42] also reported that the increase of gamma activity during the induction of anxiety was state-dependent rather than based on group differences, such as between high- and low-anxiety groups. Gamma oscillations reflect not only cortical arousal for processing emotional stimuli, but also related cognitive process including attention. Although individuals with high anxiety have a selective bias toward threatening stimuli and deploy more processing resources to threatening information [43-45], they are reported to show attentional deficits and a diffused and unstructured network related to cognitive processing $[39,46,47]$. In our study, gamma band activity had a significant negative association with the degree of anxiety. In addition, these associations were more widespread with trait anxiety than with state anxiety. Although it is speculative, these findings might reflect a deficit in maintaining attention during the resting state in individuals with high anxiety. Consistent with this, a previous study with patients with depression reported that gamma power was positively correlated with scores on inattention [48].

In the present study, the delta, alpha, theta, and beta band powers were not significantly correlated with state and trait anxiety scores. Previous studies examining EEG correlates of anxiety have reported inconsistent findings with respect to these frequency bands. A previous study reported that state and trait anxiety were positively related to alpha and negatively related to delta relative power independent of cortical site [49]. Another study reported that the absolute delta and theta powers were significantly correlated with observer ratings of anxiety in patients with panic disorder, while relative beta power was related to self-ratings of anxiety [50]. [51] found in a study of patients with social phobia that scores on trait anxiety were positively correlated with alpha power, and negatively correlated with absolute theta and slow alpha powers. In our study, delta and theta power did show a negative association with trait anxiety on most electrodes, however, the correlations did not reach significance.

This study has some limitations that need to be noted. A small sample size is the first limitation of our study which might decrease the power of our findings. In fact, despite the consistent negative association among trait anxiety and delta and theta power on most electrodes, the non-significant correlation could be due to a small sample size. In addition, a lack of a healthy comparison group limits the generalized interpretation of the present findings in the normal population as well as the differential comparison between patients with anxiety and controls. Thus, future studies with a larger sample size that include healthy controls are warranted to confirm our findings. The assessment for the level of anxiety symptoms being based on only the STAl is another limitation of our study. As aforementioned in the introduction, the symptom presentation of anxiety across the disorders (i.e., panic disorder, PTSD, and OCD) are heterogeneous [20]. As well, the association between anxiety and EEG might vary according to assessment tools (i.e., observer-rating versus self-rating) [50]. Thus, the adoption of multiple assessment tools based on a multi-informant approach may elucidate the neural correlates of anxiety. Finally, the heterogeneous constitution of participants was also a limitation. For instance, age or cognitive function may have an effect on neural correlates. Although we tried to reduce the variability with our inclusion 
criteria for age and IQ, the wide range of age and IQ in our participants might affect the results. Future studies with a strictly matched sample are warranted to confirmed our findings.

\section{Conclusions}

The present study investigated the associations between EEG power bands during resting state and the level of anxiety symptoms according to the RDoC framework, focusing on the characteristics of anxiety symptoms rather than specific diagnoses. This study identified the significantly negative correlations between the anxiety level and gamma band power in fronto-central and posterior regions. Our results provide novel and differential findings from the previous studies in that we investigated the association in resting-status, whereas the previous studies were performed on task-based situation. Future studies with a larger sample including healthy controls are needed to confirm our findings.

\section{Declarations}

\section{Ethics approval and consent to participate}

Participants gave their written informed consent after being given a complete description of the study. This study was approved by the Institutional Review Board (IRB) of the Daegu Catholic University Medical Center (DCUMC IRB approval No. CR-19-005) and was performed in accordance with the Declaration of Helsinki (World Medical Association: Ethical Principles for Medical Research Involving Human Subjects, 1964).

\section{Consent for publication}

Not applicable

\section{Availability of data and materials}

The data supporting our findings are available from the corresponding author on reasonable request. The dataset is available from f_affection@naver.com.

\section{Competing interests}

The authors have no potential conflicts of interest to disclose.

\section{Funding}

This work was supported by the grant of Research Institute of Medical Science, Daegu Catholic University (2018).

\section{Authors' contributions}

KMK - Formal analysis, Writing - original draft, Conceptualization 
SHB - Conceptualization, Data curation

JWK - Conceptualization, Formal analysis, Supervision, Designed the study

The authors read and approved the final manuscript.

\section{Acknowledgments}

Not applicable

\section{References}

1. American Psychiatric Association. Diagnostic and Statistical Manual of Mental Disorders. 5th ed. Arlington: American Psychiatric Publishing; 2013.

2. Morris SE, Cuthbert BN. Research Domain Criteria: cognitive systems, neural circuits, and dimensions of behavior. Dialogues Clin Neurosci. 2012;14(1):29-37. doi: 10.31887/DCNS.2012.14.1/smorris.

3. Kozak MJ, Cuthbert BN. The NIMH Research Domain Criteria Initiative: Background, Issues, and Pragmatics. Psychophysiology. 2016;53(3):286-97. doi: 10.1111/psyp.12518.

4. Williams LM, Goldstein-Piekarski AN, Chowdhry N, Grisanzio KA, Haug NA, Samara Z, et al. Developing a clinical translational neuroscience taxonomy for anxiety and mood disorder: protocol for the baseline-follow up Research domain criteria Anxiety and Depression ("RAD") project. BMC Psychiatry. 2016;16:68. doi: 10.1186/s12888-016-0771-3.

5. Spielberger CD. State-Trait Anger Expression Inventory. In:Weiner IB, Graighead WE, editors. The Corsini Encyclopedia of Psychology; 2010.

6. Baur V, Hänggi J, Langer N, Jäncke L. Resting-state functional and structural connectivity within an insula-amygdala route specifically index state and trait anxiety. Biol Psychiatry. 2013;73(1):85-92. doi: 10.1016/j.biopsych.2012.06.003.

7. Ursache A, Raver CC. Trait and state anxiety: relations to executive functioning in an at-risk sample. Cogn Emot. 2014;28(5):845-55. doi: 10.1080/02699931.2013.855173.

8. Prichep LS, John ER. QEEG profiles of psychiatric disorders. Brain Topogr. 1992;4(4):249-57. doi: 10.1007/BF01135562.

9. Newson JJ, Thiagarajan TC. EEG Frequency Bands in Psychiatric Disorders: A Review of Resting State Studies. Front Hum Neurosci. 2019;12:521. doi: 10.3389/fnhum.2018.00521.

10. McVoy M, Lytle S, Fulchiero E, Aebi ME, Adeleye O, Sajatovic M. A systematic review of quantitative EEG as a possible biomarker in child psychiatric disorders. Psychiatry Res. 2019;279:331-44. doi: 10.1016/j.psychres.2019.07.004.

11. Coburn KL, Lauterbach EC, Boutros NN, Black KJ, Arciniegas DB, Coffey CE. The value of quantitative electroencephalography in clinical psychiatry: a report by the Committee on Research of the American Neuropsychiatric Association. J Neuropsychiatry Clin Neurosci. 2006;18(4):460-500. doi: 10.1176/jnp.2006.18.4.460. 
12. Chabot RJ, di Michele F, Prichep L. The role of quantitative electroencephalography in child and adolescent psychiatric disorders. Child Adolesc Psychiatr Clin N Am. 2005;14(1):21-53. doi: 10.1016/j.chc.2004.07.005.

13. Perera MPN, Bailey NW, Herring SE, Fitzgerald PB. Electrophysiology of obsessive compulsive disorder: A systematic review of the electroencephalographic literature. J Anxiety Disord. 2019;62:114. doi: 10.1016/j.janxdis.2018.11.001.

14. Carvalho MR, Velasques BB, Cagy M, Marques JB, Teixeira S, Nardi AE, et al. Electroencephalographic findings in panic disorder. Trends Psychiatry Psychother. 2013;35(4):23851. doi: 10.1590/2237-6089-2013-0012.

15. Buchsbaum MS, Hazlett E, Sicotte N, Stein M, Wu J, Zetin M. Topographic EEG changes with benzodiazepine administration in generalized anxiety disorder. Biol Psychiatry. 1985;20(8):832-42. doi: 10.1016/0006-3223(85)90208-2.

16. Siciliani O, Schiavon M, Tansella M. Anxiety and EEG alpha activity in neurotic patients. Acta Psychiatr Scand. 1975;52(2):116-31. doi: 10.1111/j.1600-0447.1975.tb00028.x.

17. Taş C, Erensoy H, ibadi Y, Brown E, Tarhan N. QEEG related changes following the treatment of anxiety disorders: case series. J Neurobehav Sci. 2014;1(1):9-13. doi: 10.5455/JNBS.1392036730.

18. Perros P, Young ES, Ritson JJ, Price GW, Mann P. Power spectral EEG analysis and EEG variability in obsessive-compulsive disorder. Brain Topogr. 1992;4(3):187-92. doi: 10.1007/BF01131149.

19. Silverman JS, Loychik SG. Brain-mapping abnormalities in a family with three obsessive compulsive children. J Neuropsychiatry Clin Neurosci. 1990;2(3):319-22. doi: 10.1176/jnp.2.3.319.

20. Nitschke JB, Heller W. Distinguishing neural substrates of heterogeneity among anxiety disorders. Int Rev Neurobiol. 2005;67:1-42. doi: 10.1016/S0074-7742(05)67001-8.

21. Beck AT, Ward CH, Mendelson M, Mock J, Erbaugh J. An inventory for measuring depression. Arch Gen Psychiatry. 1961;4:561-71. doi: 10.1001/archpsyc.1961.01710120031004.

22. Sung HM, Kim JB, Park YN, Bai DS, Lee SH, Ahn HN. A study on the reliability and the validity of Korean version of the Beck Depression Inventory-II (BDI-II). J Korean Soc Bio Ther Psychiatry. 2008;14(2):201-212.

23. Kim JT, Shin DK. A study based on the standardization of the STAI for Korea. The New Medical Journal. 1978;21(11):69-75.

24. Hahn DW, Lee CH, Chon KK. Korean Adaptation of Spielberger's. STAI (K-STAI). Korean J Health Psychol. 1996;1:1-14.

25. Kim J, Lee Y, Han D, Min K, Kim D, Lee C. The utility of quantitative electroencephalography and Integrated Visual and Auditory Continuous Performance Test as auxiliary tools for the Attention Deficit Hyperactivity Disorder diagnosis. Clin Neurophysiol. 2015;126(3):532-40. doi: 10.1016/j.clinph.2014.06.034.

26. Kim JW, Kim SY, Choi JW, Kim KM, Nam SH, Min KJ, et al. Differences in Resting-state Quantitative Electroencephalography Patterns in Attention Deficit/Hyperactivity Disorder with or without 
Comorbid Symptoms. Clin Psychopharmacol Neurosci. 2017;15(2):138-45. doi: 10.9758/cpn.2017.15.2.138.

27. Kim JW, Lee YS, Han DH, Min KJ, Lee J, Lee K. Diagnostic utility of quantitative EEG in un-medicated schizophrenia. Neurosci Lett. 2015;589:126-31. doi: 10.1016/j.neulet.2014.12.064.

28. Delorme A, Makeig S. EEGLAB: an open source toolbox for analysis of single-trial EEG dynamics including independent component analysis. J Neurosci Methods. 2004;134(1):9-21. doi: 10.1016/j.jneumeth.2003.10.009.

29. Jung TP, Makeig S, Humphries C, Lee TW, McKeown MJ, Iragui V, et al. Removing electroencephalographic artifacts by blind source separation. Psychophysiology. 2000;37(2):16378.

30. Jung TP, Makeig S, Westerfield M, Townsend J, Courchesne E, Sejnowski TJ. Removal of eye activity artifacts from visual event-related potentials in normal and clinical subjects. Clin Neurophysiol. 2000;111(10):1745-58. doi: 10.1016/s1388-2457(00)00386-2.

31. Wang XJ. Neurophysiological and computational principles of cortical rhythms in cognition. Physiol Rev. 2010;90(3):1195-268. doi: 10.1152/physrev.00035.2008.

32. Buzsáki G, Wang XJ. Mechanisms of gamma oscillations. Annu Rev Neurosci. 2012;35:203-25. doi: 10.1146/annurev-neuro-062111-150444.

33. Herrmann CS, Fründ I, Lenz D. Human gamma-band activity: a review on cognitive and behavioral correlates and network models. Neurosci Biobehav Rev. 2010;34(7):981-92. doi: 10.1016/j.neubiorev.2009.09.001.

34. Tallon-Baudry C, Bertrand O. Oscillatory gamma activity in humans and its role in object representation. Trends Cogn Sci. 1999;3(4):151-162. doi: 10.1016/s1364-6613(99)01299-1.

35. Jensen 0 , Kaiser J, Lachaux JP. Human gamma-frequency oscillations associated with attention and memory. Trends Neurosci. 2007;30(7):317-24. doi: 10.1016/j.tins.2007.05.001.

36. Headley DB, Paré D. In sync: gamma oscillations and emotional memory. Front Behav Neurosci. 2013;7:170. doi: 10.3389/fnbeh.2013.00170.

37. Keil A, Müller MM, Gruber T, Wienbruch C, Stolarova M, Elbert T. Effects of emotional arousal in the cerebral hemispheres: a study of oscillatory brain activity and event-related potentials. Clin Neurophysiol. 2001;112(11):2057-68. doi: 10.1016/s1388-2457(01)00654-x.

38. Luo Q, Mitchell D, Cheng X, Mondillo K, Mccaffrey D, Holroyd T, et al. Visual awareness, emotion, and gamma band synchronization. Cereb Cortex. 2009;19(8):1896-904. doi: 10.1093/cercor/bhn216.

39. Liu J, Li J, Peng W, Feng M, Luo Y. EEG correlates of math anxiety during arithmetic problem solving: Implication for attention deficits. Neurosci Lett. 2019;703:191-197. doi: 10.1016/j.neulet.2019.03.047.

40. Schneider TR, Hipp JF, Domnick C, Carl C, Büchel C, Engel AK. Modulation of neuronal oscillatory activity in the beta- and gamma-band is associated with current individual anxiety levels. Neuroimage. 2018;178:423-434. doi: 10.1016/j.neuroimage.2018.05.059. 
41. Miskovic V, Ashbaugh AR, Santesso DL, McCabe RE, Antony MM, Schmidt LA. Frontal brain oscillations and social anxiety: a cross-frequency spectral analysis during baseline and speech anticipation. Biol Psychol. 2010;83(2):125-32. doi: 10.1016/j.biopsycho.2009.11.010.

42. Oathes DJ, Ray WJ, Yamasaki AS, Borkovec TD, Castonguay LG, Newman MG, et al. Worry, generalized anxiety disorder, and emotion: evidence from the EEG gamma band. Biol Psychol. 2008 Oct;79(2):165-70. doi: 10.1016/j.biopsycho.2008.04.005.

43. McNally R. Abnormalities in anxiety implications for cognitive neuroscience. Cognition and Emotion. 1998; 12(3):479-95. doi: 10.1080/026999398379682.

44. Aftanas LI, Pavlov SV, Reva NV, Varlamov AA. Trait anxiety impact on the EEG theta band power changes during appraisal of threatening and pleasant visual stimuli. Int J Psychophysiol. 2003;50(3):205-12. doi: 10.1016/s0167-8760(03)00156-9.

45. Derryberry $D$, Reed MA. Anxiety-related attentional biases and their regulation by attentional control. J Abnorm Psychol. 2002;111(2):225-36. doi: 10.1037//0021-843x.111.2.225.

46. Suárez-Pellicioni M, Núñez-Peña MI, Colomé A. Mathematical anxiety effects on simple arithmetic processing efficiency: an event-related potential study. Biol Psychol. 2013;94(3):517-26. doi: 10.1016/j.biopsycho.2013.09.012.

47. Klados MA, Paraskevopoulos E, Pandria N, Bamidis PD. The impact of math anxiety on working memory: A cortical activations and cortical functional connectivity EEG study. IEEE Access. 2019;7:15027-15039. doi: 10.1109/ACCESS.2019.2892808.

48. Roh SC, Park EJ, Shim M, Lee SH. EEG beta and low gamma power correlates with inattention in patients with major depressive disorder. J Affect Disord. 2016;204:124-30. doi:

10.1016/j.jad.2016.06.033.

49. Knyazev GG, Savostyanov AN, Levin EA. Alpha oscillations as a correlate of trait anxiety. Int J Psychophysiol. 2004;53(2):147-60. doi: 10.1016/j.jpsycho.2004.03.001.

50. Knott VJ, Bakish D, Lusk S, Barkely J, Perugini M. Quantitative EEG correlates of panic disorder. Psychiatry Res. 1996;68(1):31-9. doi: 10.1016/s0925-4927(96)02962-9.

51. Sachs G, Anderer P, Dantendorfer K, Saletu B. EEG mapping in patients with social phobia. Psychiatry Res. 2004;131(3):237-47. doi: 10.1016/j.pscychresns.2003.08.007.

\section{Figures}




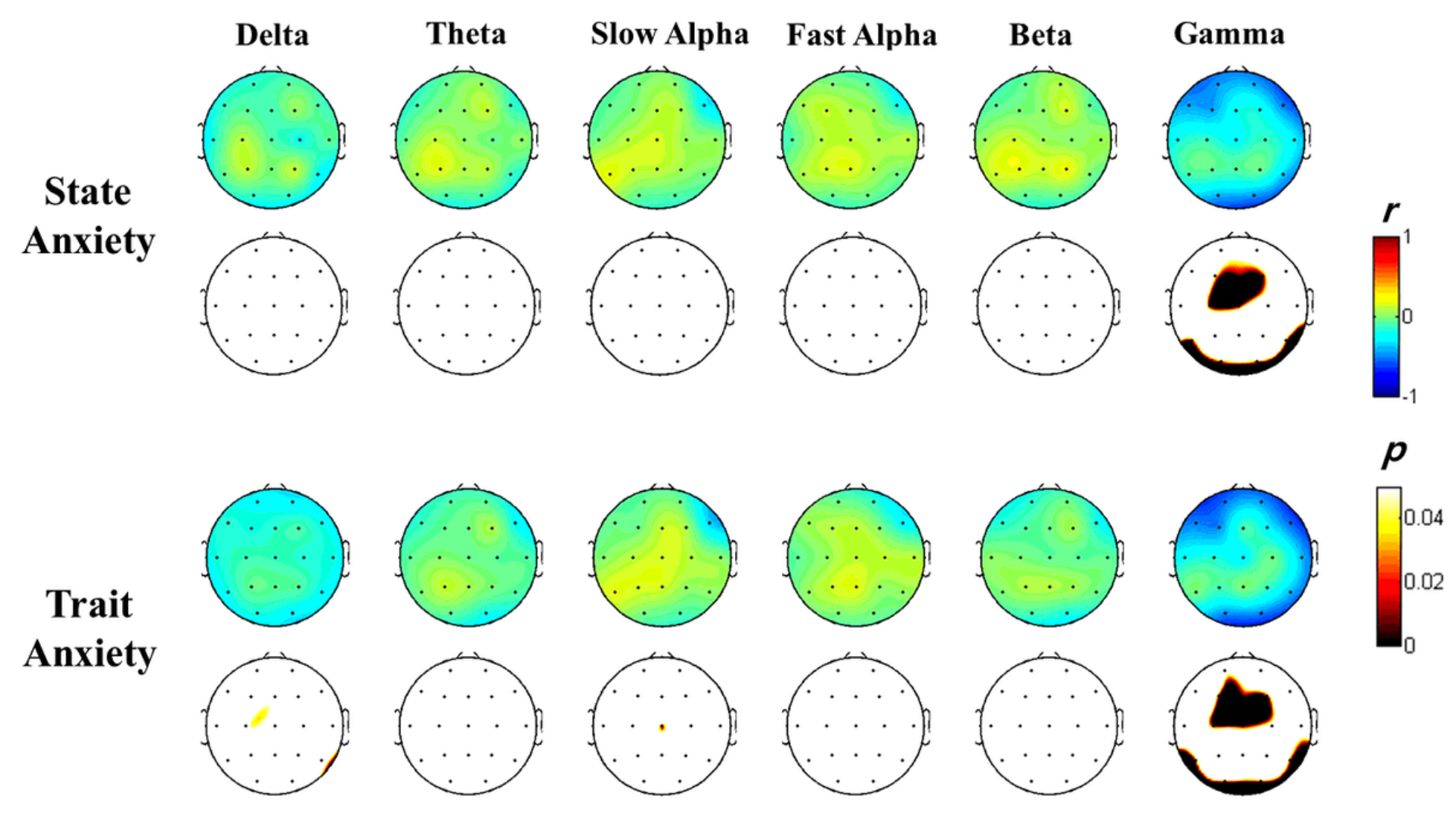

Figure 1

Topographical representation of the statistical results of the Pearson's partial correlation analysis controlled for age and gender between the anxiety symptom and EEG analysis. Upper topoplots denote Pearson's correlation coefficients $(R)$. Lower topoplots denote statistical significance $(P)$ after applying the Bonferroni correction; EEG, electroencephalogram 